\title{
Measurement of Dynamic Portfolio VaR Based on Mixed Vine Copula Model
}

\author{
Zhao Ru-bo, Tian Yi-xiang, Tian Wei, Chen Xiu-rong \\ School of Management and Economics, University of Electronic Science and Technology of China, Chengdu, China
}

Email address:

zhaoruboecon@126.com (Zhao Ru-bo), tianyx@uestc.edu.cn (Tian Yi-xiang),12240455@qq.com (Tian Wei), 465123149@qq.com (Chen Xiu-rong)

\section{To cite this article:}

Zhao Ru-bo, Tian Yi-xiang, Tian Wei, Chen Xiu-rong. Measurement of Dynamic Portfolio VaR Based on Mixed Vine Copula Model. Journal of Finance and Accounting. Vol. 5, No. 2, 2017, pp. 80-86. doi: 10.11648/j.jfa.20170502.12

Received: March 17, 2017; Accepted: April 14, 2017; Published: April 21, 2017

\begin{abstract}
The measurement of portfolio VaR has been a hot issue in the field of the academic and the industry. This paper applies three kinds of Vine Copula model to describe high-dimensional dependency structure between multiple assets, introduces mixed binary copula function to improve the accuracy of tail dependence structure. We use six important stock markets as stock portfolio to test this model. The empirical results show that introducing mixed Copula function can improve the measurement reliability of Vine Copula model, and the reliability of mixed R-Vine model is highest in three kinds of mixed Vine Copula models.
\end{abstract}

Keywords: Mixed Copula, Vine Copula, Dynamic of VaR, Portfolio

\section{Introduction}

Financial risk management has always been a hot and difficult issue for investors, government financial management and financial academia [1]. Especially in recent years, the outbreaks of global risk crisis highlight the importance of financial risk management and urgency. In financial risk management, the most critical job is how to accurately measure financial risks. Only measuring financial risks accurately can we ensure the effectiveness of risk management. At present, the mainstream approach in a single asset or portfolio risk measure is VaR (Value at Risk) [2]. Measuring the VaR of a single asset is simpler, but because the financial institution and the investor tend to invest in the form of a portfolio, and to measure the VaR of the portfolio, it must first depict the high-dimensional structure of the portfolio. Therefore, how to accurately characterize the interdependence between the portfolio will be the key to measuring the portfolio VaR.

Many scholars have shown that financial assets have many typical characteristics such as spikes and fat tail, non-normality, volatility aggregation and leverage. At the same time, the dependent structure between financial assets is non-linear [3]. Therefore, the linear correlation model, Granger causality test and other linear model cannot accurately describe the non-linear dependent structure of financial assets [4]. And then based on the above model is difficult to accurately measure the portfolio VaR. Copula function effectively overcomes the shortcomings of the above linear model, not only can describe the nonlinear dependency structure between financial assets, but also can include typical fact characteristics into the study constraints. Since then, Copula function has been widely used by scholars in the fields of financial risk management and risk measurement, and has achieved many important practical achievements.

At present, Scholars use binary copula functions in the financial research. When the number of variables is more than two, the binary copula function will face dimension curse. Although the multidimensional Copula function is used to solve the problem of high-dimensional dependency structure, the multivariate Copula function assumes that the dependent structure between different financial assets is same, and this is not consistent with the actual situation, so that the multivariate Copula function cannot accurately depict the interdependent structure between multiple assets [5].

Recently, the Vine Copula model has effectively solved the problem of high-dimensional dependency structure of multiple financial assets, and vine copula function has more 
flexibility than multivariate Copula function. Joe, Bedford and Cooke have made groundbreaking work for the construction of the Vine Copula model [6] [7] [8]. Vine copula model is layered to depict the dependent structure, and the model greatly reduce the difficulty of characterizing high-dimensional dependent structure. In addition, the Vine Copula model can be used to select the appropriate binary Copula function according to the different characteristics of the object-dependent structure, so that the Vine Copula model has more flexibility and practicability in characterizing the high-dimensional dependency structure. Bedford and Cooke proposed R-Vine, R-Vine Copula is more flexible and complex, the difficulty of estimation is relatively large. The application is far less than its two special circumstances: C-Vine and D-Vine [8] [9]. Therefore, to highlight the flexibility and advantage of R-Vine model in characterizing the structure of high-dimensional dependencies, this paper not only uses C-Vine and D-Vine, but also uses R-Vine.

In the use of vine copula model to describe high-dimensional dependent structure, they chose binary copula function based on the AIC and other indicators. Although a relatively optimal Vine Copula model can be selected, this will increase the risk of Vine Copula's model. In addition, a single binary Copula function is usually only able to describe some of the tail dependent structure or cannot describe the tail dependent structure, and tail dependent structure represents the degree of financial assets in extreme cases, the tail-dependent structure is of great importance to accurately measure the extreme financial risks of financial assets. Clayton, Frank and Gumbel can characterize the lower end dependent structure, symmetric dependent structure and upper tail dependent structure [10]. To make full use of the characteristics of Copula function, this paper construct a mixed copula function to describe the dependent structure of two-dimensional variables in the vine Copula model.

Based on the above analysis and understanding, this paper will take the world's six major stock markets as the study object, using the ARMA-GJR-t model to capture the important typical factual characteristics of the yield and volatility of financial assets; aiming at the complex high-dimensional dependency structure between the portfolio, three Vine Copula models are introduced, At the same time, the mixed Copula function is used in the Vine Copula model to describe the asymmetric tail dependent structure between the binary variables, in order to carry out the VaR dynamic risk measure; And then use the rigorous likelihood ratio test to test the reliability of the stock market portfolio risk model. And tries to prove the following two conclusions through the relevant theoretical analysis and empirical research: The $\mathrm{R}$-Vine Copula model is more reliable than the $\mathrm{C}$-Vine and D-Vine Copula models in the risk measure. The hybrid Copula function can improve the reliability of the Vine Copula model for the dynamic risk measure.

So far, many scholars have used a variety of methods to carry out a lot of research on portfolio risk measurement, and achieved fruitful research results. Aloui et al. used the binary Copula function to study the financial risk of the financial crisis between the BRIC countries and the US stock market, and measured the risk of the binary portfolio [11]. Deng et al. used the Pair Copula model to measure portfolio risk, while also using Mean-CVaR to optimize the portfolio [12]. Zhou et al used multiple Copula function to measure the risk of multiple financial portfolio [13]. Brechmann et al. used the $\mathrm{R}$-Vine Copula model to predict the portfolio risk value. The results show that the R-Vine model measures better than the DCC model [14]. Weiß et al. used the Vine Copula model to predict the portfolio risk. The results show that the Vine Copula model can accurately measure the risk of portfolio [15]. Fan Guobin et al. used the C-Vine Copula model to characterize the nonlinear dependencies between multiple financial assets. The empirical results show that the C-Vine Copula model can describe the complex tail-dependent structure of financial assets more accurately than the traditional multi-Copula function [16]. Gao used the vine Copula model to measure the risk of multiple assets [17]. Zhang et al. used the R-Vine, C-Vine and D-Vine models to explore the interdependent structure of different financial assets in China. The empirical results show that China's financial market shows thick-tailed correlation and asymmetry [18]. Ma Feng used the Vine Copula model to carry out dynamic risk forecasting of the stock market portfolio [19]. Zhao $\mathrm{Lu}$ used the multivariate t-Copula function to measure the risk of the energy portfolio and optimize the weight of the portfolio [20].

Although these scholars have achieved satisfactory results in the relevant studies, these studies still have the following two shortcomings: these scholars use only multiple elements to describe the same dependent structure of the Copula function, but between financial assets Dependent structure may not be completely consistent, then the use of multiple Copula function will be questionable; Although there are a few scholars using Vine Copula function to describe high-dimensional dependent structure, but they used binary copula function in vine copula model, and these binary Copula function cannot describe the asymmetric tail dependent structure. This article not only uses C-Vine, D-Vine, but also uses more flexible and complex R-Vine in characterizing the portfolio structure. In addition, the hybrid Copula function is introduced into the Vine Copula model, and a new mixed Vine Copula model is constructed. Therefore, compared with other scholars, the hybrid vine copula can measure the dependent structure more accurate especially in the financial crisis, which has a prominent advantage in measuring the dynamic risk of the portfolio.

The logical structure of this paper is roughly arranged as follows, the following is the second part of this article, constructing the dynamic VaR measure model based on the mixed vine copula model. The third part uses the constructed risk forecasting model to carry on the dynamic risk measure to the portfolio, the last part is the conclusion. 


\section{Construction of Dynamic Risk Measurement Model of Portfolio}

\subsection{Vine Copula Model}

Sklar theorem suggests that the d-dimensional cumulative distribution function $F_{i}$ can be decomposed into an edge distribution function and a Copula Function $\mathrm{C}$, which can be expressed as [21]:

$$
F\left(X_{1}, X_{2}, \cdots, X_{d}\right)=C\left(F_{1}\left(x_{1}\right), \cdots, F_{d}\left(x_{d}\right)\right.
$$

The corresponding joint density function can be expressed as:

$$
f\left(x_{1}, x_{2}, \cdots, x_{d}\right)=c\left(F_{1}\left(x_{1}\right), \cdots, F_{d}\left(x_{d}\right)\right) \sum_{i=1}^{d} f_{i}\left(x_{i}\right)
$$

Where $\mathrm{c}$ is the Copula function density function and $f_{i}$ is the edge density function.

As the number of variables increases, it is increasingly complicated to measure the joint distribution between these variables. Joe, Bedford and Cooke proposed the Vine Copula model, the high-dimensional joint density function is decomposed into several binary joint distribution density functions and corresponding edge density functions, so that the joint distribution of multivariate variables is simplified. there are a variety of logical structure in the vine copula model decomposition, Bedford et al. introduced the vine to describe these decomposition structure. The commonly used decomposition structures include Canonical Vine (C-Vine), Drawable Vine (D-Vine) and Regular Vine (R-Vine) [22]. The structure of C-Vine and D-Vine is more fixed and simple than $\mathrm{R}$-Vine, and the structure of R-Vine is more flexible.

A $d$-dimensional C-Vine or D-Vine decomposition structure can be represented by a $d-1$ tree, where the $\mathrm{j}$-th tree has $d+1-\mathrm{j}$ nodes, $d$-j edges, and each edge corresponds to a binary Copula function.

The edge of the tree $T_{j}$ becomes a node in the tree $T_{j+1}$. For the d-dimensional joint density function can be decomposed into the $d(d-1) / 2$ edge and the corresponding edge density function according to $\mathrm{C}$-Vine and $\mathrm{D}$-Vine. The $\mathrm{C}$-Vine and D-Vine density functions can be expressed as follows:

$$
\begin{gathered}
f\left(x_{1}, \cdots, x_{d}\right)=\sum_{k=1}^{d} f_{k}\left(x_{k}\right) \sum_{j=1}^{d-1} \sum_{i=1}^{d-j} c_{i, i+1 \mid i+1, \cdots i+j-1}\left(F\left(x_{i} \mid x_{i+1}, \cdots, x_{i+j-1}\right), F\left(x_{i+j} \mid x_{i+1}, \cdots, x_{i+j-1}\right)\right) \\
f\left(x_{1}, \cdots, x_{d}\right)=\sum_{k=1}^{d} f_{k}\left(x_{k}\right) \sum_{j=1}^{d-1} \sum_{i=1}^{d-j} c_{j, j+i \mid 1, \cdots, j-1}\left(F\left(x_{j} \mid x_{1}, \cdots, x_{j-1}\right), F\left(x_{j+i} \mid x_{1}, \cdots, x_{j-1}\right)\right)
\end{gathered}
$$

Where $i$ is the hierarchy of the tree, and $j$ is the edge of each tree.

Compared with C-Vine and D-Vine, the decomposition structure of R-Vine is not fixed, So R-vine is relatively more flexible. The density function of R-Vine is:

$$
f\left(x_{1}, \cdots, x_{d}\right)=\sum_{k=1}^{d} f_{k}\left(x_{k}\right) \sum_{k=1}^{d-1} \sum_{i=k+1}^{d} c_{m_{k, k}, m_{i, k} \mid m_{i+1, k}, \cdots, m_{n, k}}\left(F_{m_{k, k} \mid m_{i+1, k}, \cdots, m_{n, k}}, F_{m_{i, k} \mid m_{i+1, k}, \cdots, m_{n, k}}\right)
$$

\subsection{Mixed Vine Copula Model Construction and Parameter Estimation}

When using the Vine Copula model studies the $d$-dimensional financial asset-dependent structure, except that the appropriate decomposition structure is selected, it is also necessary to further determine the binary Copula function in the decomposition structure. At present, in the relevant research, scholars often in terms of AIC and BIC. The binary Copula functions in the decomposed structure are selected one by one from the binary Copula functions such as Gauss and t-Copula. Gumbel, Clayton and Frank constitute a hybrid Copula function just to accurately measure the asymmetric tail dependent structure, Therefore, this paper will use the hybrid Copula function in the Vine Copula model to describe the binary variable dependent structure.

The mixed Copula function distribution function is shown below:

$$
C(u, v ; \Psi)=\pi_{1} C_{c}(u, v ; \theta)+\pi_{2} C_{f}(u, v ; \lambda)+C_{g}(u, v ; \alpha)
$$

where $C_{c}$ is Clayton function, $C_{f}$ is rank function, $C_{g}$ is Gumbel function, $\Psi$ is the parameter to be estimated, $\omega_{i}, i=1,2,3$ is weight of three Copula functions, $\sum^{3} \omega_{i}=1$.

this paper will use the maximum likelihood parameter estimation method to estimate the mixed Vine Copula model parameters, the specific estimation process is as follows:

1) To sort the nodes in the decomposition structure. For the C-Vine decomposition structure, this paper determines the order of the nodes based on the sum of the Kendall coefficients of each tree. When the order of the nodes in the first layer tree is determined, the nodes in the remaining trees will be fixed. In this paper, the D-Vine node ordering will be determined based on Brechmann's method [22]. R-Vine is more flexible than C-Vine and $\mathrm{D}$-Vine, and it is necessary to first find the optimal R-Vine matrix (RVM) to determine the order of the nodes. In this paper, we refer to the "maximum spanning tree" method proposed by Brecchmann to select the 
appropriate RVM, and then determine the arrangement of nodes in R-Vine decomposition based on RVM content.

2) Estimate the parameters of each edge in tree $j$. Based on the decomposed structure that has been determined, the parameters of the binary mixed Copula function represented by each edge are estimated using the maximum likelihood estimation method, and the corresponding conditional distribution function. R-Vine model conditional distribution function needs to be determined according to the decomposition structure.

3 ) The Copula function parameters for each edge on tree $j+1$ are estimated based on the calculated conditional distribution function until the Copula function parameters are mixed for each edge of all trees.

\subsection{Marginal Distribution Model}

As the stock market returns often have fat tail, non-normal, autocorrelation, volatility asymmetry and other characteristics, Therefore, this paper chooses the student $\mathrm{t}$ distribution to describe the distribution characteristics of the stock market returns. The ARMA $(1,1)-\operatorname{GJR}(1,1)-t$ model is used to characterize the autocorrelation, heteroskedasticity and fluctuation asymmetry of stock market returns. marginal distribution model is as follows:

$$
\begin{gathered}
R_{n t}=u_{n}+\phi_{n} R_{n, t-1}+\theta_{n} \varepsilon_{n, t-1}+\varepsilon_{n t} \\
\varepsilon_{n t}=\sigma_{n t} \xi_{n t} \\
\sigma_{n t}^{2}=\omega_{n}+\beta_{n} \sigma_{n, t-1}^{2}+\alpha_{n} \varepsilon_{n, t-1}^{2}+\gamma_{n} \varepsilon_{n, t-1}^{2} I\left(\varepsilon_{n, t-1}<0\right)
\end{gathered}
$$

Where $\sigma_{n t}^{2}$ is conditional variance, $\xi_{n t}$ is standard residual and obey the standard student $\mathrm{t}$ distribution. $I(\cdot)$ is the instruction function. When the condition is satisfied, the value is 1 , otherwise it is 0 .

\subsection{Portfolio Dynamic VaR Measure}

At present, the risk management mainly uses VaR (Value at Risk) to measure, VaR measures the maximum possible loss of a portfolio at a given confidence level for a certain period in the future. The mathematical expression is as follows:

$$
P\left(\sum_{i=1}^{d} \omega_{i} X_{i} \leq \operatorname{VaR}_{t}(\alpha) \mid \Omega_{t-1}\right)=\alpha
$$

Where $d$ is number of assets, $\omega_{i}$ is the weight of the i-th asset $\sum_{i=1}^{d} \omega_{i}=1$.

As direct estimate of portfolio VaR is very difficult, most scholars use Monte Carlo simulation method.

Therefore, this article will also use Monte Carlo simulation method, the specific steps are as follows:
(1) Use ARMA $(1,1)$-GJR $(1,1)$-t to estimate the yield of each asset in the portfolio, Extracting the volatility $\sigma_{i, t}$ of each stock market conditions. On this basis, the standard yield series is transformed by probability integral, and the uniform distribution sequence of $i . i . d(0,1)$ is obtained, expressed as Udata;

(2) Using the resulting sequence Udata, the nodes of R-Vine, C-Vine and D-Vine are sorted first. Then the parameters of the three mixed Vine Copula models are estimated by using the maximum likelihood estimation method. Monte Carlo simulation based on the estimated model parameters was simulated 5000 times per day, and finally the $T \times 5000 \times 6$ dimension array was obtained.

(3) The simulation array is inversed based on student $t$ distribution. The distribution of the residual value $\left(z_{1, t}, \cdots, z_{6, t}\right)$ of the combined assets is obtained, and then the distribution of the daily returns of the combined assets is obtained according to the formula (7).

$$
X_{t}=\sum_{i=1}^{6} \omega_{i}\left(u_{i, t}+\sigma_{i, t} z_{i, t}\right)
$$

(4) Finally, according to Equation 10, we can get the dynamic VaR value of the portfolio every day during the research period.

\section{Empirical Results and Analysis}

\subsection{Sample Selection}

In this paper, the Shanghai Composite Index (SSEC), the Hang Seng Index (HSI), the Nikkei 225 Index (N225), the Frankfurt DAX Index (DAX), the London Financial Times 100 Index (FTSE100), the S \& P 500 Index (S \& P500) are taken as important stock market representatives. The study sample is the daily closing price from January 3, 2012 to December 31, 2015. The data comes from the Resset database. As each stock market trading time is not the same, to maintain the six stock market yields data length consistent, this paper eliminates the inconsistency of each stock trading data, and finally the sample number is 711 .

As can be seen from the descriptive statistical analysis results in Table 1, The six stock market sequences show significant "spikes" and "fat tail" characteristics, and do not obey the normal distribution assumptions, The J-B test results also confirm the non-normality of the stock market income sequence. In addition, the ARCH effect test statistic LM indicates that the six stock markets have a significant ARCH effect, that is, the six stock market sequences have fluctuating aggregation effect and fluctuating time-varying characteristics. 
Table 1. The results of descriptive statistical analysis of log yields in each stock market.

\begin{tabular}{|c|c|c|c|c|c|c|c|c|}
\hline & Mean & Std & Skewness & Kurtosis & J-B & LM(5) & LM(10) & LM (15) \\
\hline SSEC & 0.0143 & 1.0060 & $-0.0404^{* * *}$ & $4.9705^{* * *}$ & $115.2172^{* * *}$ & $49.6730^{* * *}$ & $50.1325^{* * *}$ & $70.7780^{* * *}$ \\
\hline HSI & 0.0694 & 1.9499 & $-1.1909^{* * *}$ & $12.7604^{* * *}$ & $2990.3^{* * *}$ & $38.9607^{* * *}$ & $51.2324^{* * *}$ & $53.8874^{* * *}$ \\
\hline N225 & 0.0219 & 1.2358 & $0.0256^{* * *}$ & $6.0380^{* * *}$ & $273.4957^{* * *}$ & $43.8700^{* * *}$ & $48.6460^{* * *}$ & $77.3747^{* * *}$ \\
\hline DAX & 0.1124 & 1.5652 & $-0.5343^{* *}$ & $5.9404^{* * *}$ & $289.9660^{* * *}$ & $31.1976^{* * *}$ & $37.1481^{* * *}$ & $51.2971^{* * *}$ \\
\hline FTSE100 & 0.0793 & 1.3308 & $-0.2467^{* * *}$ & $4.2661^{* * *}$ & $54.7002^{* * *}$ & $29.4465^{* * *}$ & $32.1903^{* * *}$ & $40.3854^{* * *}$ \\
\hline S\&P500 & 0.0685 & 0.9767 & $-0.5133^{* * *}$ & $9.5420^{* * *}$ & $1299.1^{* * *}$ & $38.9313^{* * *}$ & $39.9355^{* * *}$ & $51.8063^{* * *}$ \\
\hline
\end{tabular}

Note: $* * * * *$, respectively, at $1 \%, 5 \%$ significant level of significance, J-B t is Jarque-Bera statistics, LM is Engle's ARCH test statistics (brackets indicate the lag order).

\subsection{Marginal Distribution Model Estimation Results}

Table 2. Parameter of marginal distribution model.

\begin{tabular}{lllllll}
\hline & SSEC & HSI & N225 & DAX & FTSE100 & S\&P500 \\
\hline$u$ & 0.0301 & 0.0342 & 0.0738 & 0.0427 & 0.0969 & 0.1551 \\
$\phi$ & -0.7617 & -0.7618 & -0.8606 & 0.7082 & 0.0001 & -0.8433 \\
$\theta$ & 0.7958 & 0.7818 & 0.8860 & -0.6965 & 0.0209 & 0.8151 \\
$\omega$ & 0.0954 & 0.0721 & 0.0833 & 0.2963 & 0.0421 & 0.0881 \\
$\beta$ & 0.7645 & 0.9217 & 0.8889 & 0.7485 & 0.905 & 0.7216 \\
$\alpha$ & 0.0621 & 0.0563 & 0.0000 & 0.0126 & 0.0354 & 0.0021 \\
$\gamma$ & 0.3182 & 0.0131 & 0.1080 & 0.2475 & 0.1501 & 0.4084 \\
$\nu$ & 4.9712 & 3.8751 & 5.9664 & 5.8737 & 5.4258 & 5.2567 \\
$\mathrm{Q}(5)$ & 0.3297 & 0.6279 & 0.3541 & 0.6432 & 0.8998 & 0.8997 \\
$\mathrm{~K}-\mathrm{S}$ & 0.4342 & 0.4944 & 0.8165 & 0.9921 & 0.9091 & 0.7123 \\
\hline
\end{tabular}

Note: K-S is Kolmogorov-Smirnov test P value.

As there are non-normality, spikes and fat tail and other characteristics in the six stock market returns sequence. In this paper, the $\operatorname{ARMA}(1,1)-\operatorname{GJR}(1,1)$-t model is used to analyze the marginal distribution of the six stock markets. The parameters result of the marginal distribution model is shown in Table 2. And then extract the stock market standard rate of return sequence, perform the probability integral transformation. To test the fitting ability of the marginal distribution model for the six stock market return series, ensuring that the series after the probability integral transformation is uniformly distributed by i. i. d $(0,1)$. We also used the Kolmogorov-Smirnov (K-S) test, which showed that the series is uniformly distributed. In addition, we also used the Ljung-Box Q test, the test results show that the stock market series does not exist autocorrelation after the integral transformation. Therefore, the marginal distribution model ARMA $(1,1)$-GJR $(1,1)$-t can describe the six stock market returns series. On this basis, the Vine Copula model can be used to analyze the interdependent structure between the six stock markets, measure the dynamic risk of portfolio.

\subsection{Portfolio Risk Measurement Model Reliability Test}

As this paper taken the world's six major stock market as research object, at the same time this paper uses mixed Copula function in the Vine Copula model. Therefore, whether it is $\mathrm{C}$-Vine, D-Vine or R-Vine, the estimated parameters are up to 80 , considering the article space constraints, here we no longer give three types of rattan structure.

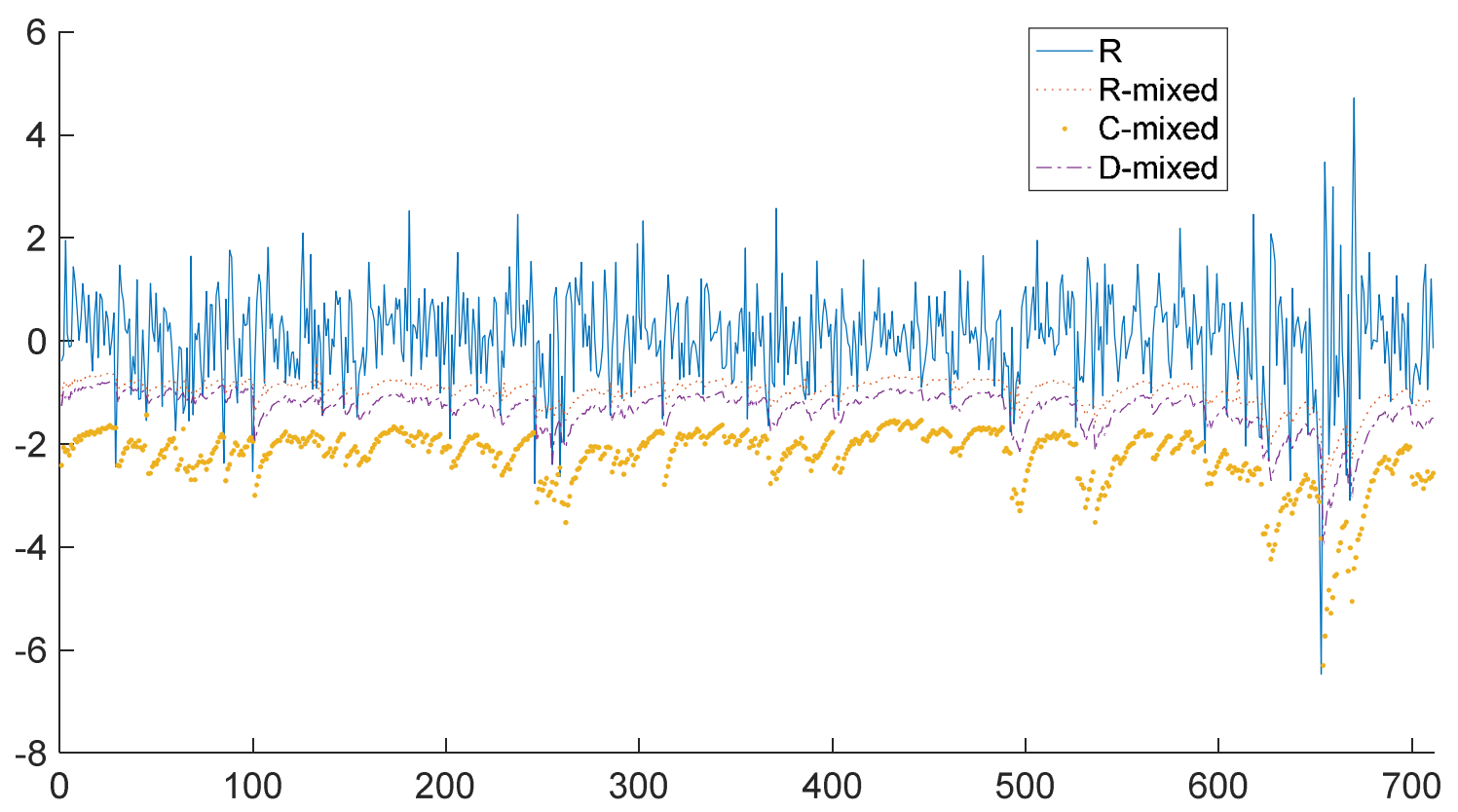

Figure 1. Stock market portfolio VaR. 
Figure 1 shows the VaR measurement results for the stock market portfolio based on the mixed C-Vine, D-Vine and R-Vine models at the $5 \%$ quantile level during the sample period $^{1}$. It can be seen from Figure 1 that the three mixed Vine Copula models can accurately measure the dynamic risk of the stock market portfolio.

After measuring the dynamic risk of the stock market portfolio through different models, an important job is to test the reliability of the risk measurement model. In this paper, we will use the conditional coverage LR test proposed by Christoffersen (1998) [23], because the conditional coverage LR test considers the failure number and independence, so the test has a more comprehensive. To be able to compare the differences between the mixed Vine Copula model and the Vine Copula model in the portfolio risk measure, we will backtest three Vine Copula models. The backtesting results are shown in table 3 under the same weight conditions.

Table 3. Portfolio Risk Measurements Back-testing.

\begin{tabular}{llllccc}
\hline & $\mathbf{1 \%}$ & \multicolumn{3}{c}{$\mathbf{5 \%}$} & $\mathbf{1 0} \%$ \\
\hline & $\mathbf{K}(\mathbf{E})$ & $\boldsymbol{L R}_{c c}$ & $\mathbf{K 1}(\mathbf{K} \mathbf{)}$ & $\boldsymbol{L R}_{c c}$ & $\mathbf{K 1}(\mathbf{K})$ & $\boldsymbol{L} \boldsymbol{R}_{c c}$ \\
\hline C-mixed & $6(7.11)$ & 0.6672 & $34(35.55)$ & 0.2364 & $72(71.1)$ & 0.5636 \\
D-mixed & $6(7.11)$ & 0.6672 & $37(35.55)$ & 0.3657 & $70(71.1)$ & 0.8932 \\
R-mixed & $7(7.11)$ & 0.9668 & $36(35.55)$ & 0.5645 & $74(71.1)$ & 0.9254 \\
R-Vine & $8(7.11)$ & 0.1901 & $41(35.55)$ & 0.1800 & $72(71.1)$ & 0.3523 \\
C-Vine & $10(7.11)$ & 0.1817 & $40(35.55)$ & 0.4003 & $73(71.1)$ & 0.3911 \\
D-Vine & $7(7.11)$ & 0.1534 & $40(35.55)$ & 0.4003 & $72(71.1)$ & 0.3523 \\
\hline
\end{tabular}

Note: $\mathrm{K}$ and $\mathrm{E}$ represent the actual failure of VaR measure and the number of theoretical failures, respectively, for the conditional coverage test $\mathrm{P}$ value, The higher the $\mathrm{P}$ value, the higher the reliability of the model.

It can be seen from Table 3 that the reliability of the three mixed Vine Copula models is higher than three Vine Copula models at three confidence levels, which shows that the binary hybrid Copula function can more accurately describe the interdependence structure between binary financial assets, especially the extremely dependent structure between financial assets. Finally, the mixed Vine Copula model is more accurate and reliable in measuring the dynamic risk of a portfolio asset. In addition, we can see that the reliability of the mixed R-Vine model is higher than the mixed C-Vine and D-Vine model, which shows that the mixed R-Vine model is more accurate in describing the dependent structure of multicomponent financial assets than mixed $\mathrm{C}$-Vine and mixed D-Vine models.

Since the equal weight is a very special case. To ensure the robustness of the test results in this paper, we refer to the Mean-CVaR method to optimize the weight of the portfolio and yield a new portfolio weight. The new weights for the portfolio are: $0.5223,0.1081,0.02,0.0199,0.1441,0.1856$. And then backtest again, the result is shown in Table 4. It can be seen from Table 4 that the test results not only prove the reliability of the backtesting result under the same weight condition, but also further prove the superiority of the mixed

1. To be able to observe the VaR measure of the combined asset clearly, we only report the results of the VaR measure under the condition of $5 \%$ quantile and three mixed Vine Copula models.
Vine Copula model, especially the mixed R-Vine Copula model in measuring the dynamic risk of the portfolio assets.

Table 4. Portfolio Risk Measurements Back-testing.

\begin{tabular}{lllllll}
\hline & $\mathbf{1 \%}$ & \multicolumn{3}{c}{$\mathbf{5 \%}$} & \multicolumn{3}{l}{$\mathbf{1 0 \%}$} \\
\hline & $\mathbf{K 1}(\mathbf{K})$ & $\boldsymbol{L R}_{c c}$ & $\mathbf{K 1}(\mathbf{K 2})$ & $\boldsymbol{L R}_{c c}$ & $\mathbf{K 1}(\mathbf{K 2})$ & $\boldsymbol{L R}_{c c}$ \\
\hline C-mixed & $6(7.11)$ & 0.6672 & $43(35.55)$ & 0.3187 & $73(71.1)$ & 0.3911 \\
D-mixed & $6(7.11)$ & 0.6672 & $35(35.55)$ & 0.9728 & $56(71.1)$ & 0.0354 \\
R-mixed & $8(7.11)$ & 0.7422 & $34(35.55)$ & 0.9231 & $70(71.1)$ & 0.1360 \\
R-Vine & $10(7.11)$ & 0.1817 & $46(35.55)$ & 0.1162 & $79(71.1)$ & 0.0107 \\
C-Vine & $14(7.11)$ & 0.0395 & $46(35.55)$ & 0.1162 & $80(71.1)$ & 0.0124 \\
D-Vine & $10(7.11)$ & 0.1817 & $46(35.55)$ & 0.1162 & $79(71.1)$ & 0.0107 \\
\hline
\end{tabular}

Note: $\mathrm{K}$ and $\mathrm{E}$ represent the actual failure of VaR measure and the number of theoretical failures, respectively, for the conditional coverage test $\mathrm{P}$ value, The higher the $\mathrm{P}$ value, the higher the reliability of the model.

\section{Conclusion}

It is a hot topic to study the high-dimensional dependency structure among many financial assets, especially the tail-dependent structure of financial assets during the financial crisis. Therefore, we introduce a more flexible and accurate Vine Copula model, and we use the mixed Copula function composed of Clayton, Frank and Gumbel to describe the binary dependency structure to measure asymmetric tail dependent structure between financial assets accurately. And taking the world's six major stock markets as research object, using Monte Carlo simulation technology, applying three mixed vine Copula model to measure the dynamic risk of stock portfolio, carrying out a rigorous Back-testing. The empirical results show that the reliability of the three mixed Vine Copula models is significantly better than other three types of Vine Copula models. In addition, the reliability of the mixed R-Vine model is superior to the mixed C-Vine and mixed D-Vine models, which shows that the mixed R-Vine model can measure the stock portfolio dependence structure accurately.

\section{Acknowledgement}

The author would like to thank the National Social Science Fund of the People's Republic of China for financially supporting this study under Contract No. 14BJY174.

\section{References}

[1] Lin Yu, "Measuring Dynamic Risk of Financial Markets Based on Stylized Facts and Extreme Value Theory," Investment Research, vol. 31, pp. 41-56, 2012.

[2] Wang peng, "Calculating VaR and ES based on volatility models with time-varying higher-moments," Journal of Management Sciences in China, vol.16, pp.33-45, 2013.

[3] J. L. Wu, G. Chen, and C. Huang, "Long-term dynamic trends in tail dependence of Chinese A, B and $\mathrm{H}$ stock markets: Empirical analysis based on multi-regime smoothing transition mixed copula model," Journal of Management Sciences in China, vol. 18, pp. 50-65, 2015. 
[4] J. L. Wu and E. H. Zhang, "Subprime mortgage crisis, market risk and stock market interdependence," World Economic, vol. 3, pp.95-108, 2010.

[5] W. W. Guo and M. Zhong, "An empirical study on dependency structure and risk measure of style portfolio in Chinese stock market based on vine copula model," Manage Review, vol.25, pp.41-52, 2013.

[6] H. Joe, "Families of m-variate distributions with given margins and $\mathrm{m}(\mathrm{m}-1) / 2$ bivariate dependence parameters," Lecture Notes-Monograph Series, pp.120-141, 1996.

[7] H. Joe, "Multivariate models and multivariate dependence concepts," CRC Press, 1997.

[8] T. Bedford and R. M. Cooke, "Probability Density Decomposition for Conditionally Dependent Random Variables Modeled by Vines," Annals of Mathematics and Artificial Intelligence, vol. 32, pp. 245-268, 2001.

[9] T. Bedford and R. M. Cooke, "Vines: A New Graphical Model for Dependent Random Variables," Annals of Statistics, vol. 30, pp. 1031-1068, 2002.

[10] Y. H. Wei and S. T. Qi, "A Study on the Crisis of Financial Crisis in Emerging Markets in Asia - A Method Based on Copula 's Theory," International Finance Research, vol.9, pp. 22-29, 2008.

[11] R. Aloui, M. S. B. Aïssa and D. K. Nguyen, "Global financial crisis, extreme interdependences, and contagion effects: The role of economic structure?" Journal of Banking \& Finance, vol.35, pp.130-141, 2010.

[12] L. Deng, C. Ma and W. Yang, "Portfolio optimization via pair copula-GARCH-EVT-CVaR model," Systems Engineering Procedia, vol.2, pp. 171-181, 2011.

[13] X. H. Zhou, B. S. Zhang and Y. W. Dong, "Risk measurement of financial portfolio based on Copula-SV-GPD model," Journal of Management Sciences in China, vol. 15, pp.70-78, 2012.
[14] E. C. Brechmann and C. Czado, "Risk management with high-dimensional vine copulas: An analysis of the Euro Stoxx 50,” Statistics \& Risk Modeling, vol.30, pp.307-342, 2013.

[15] G. N. F. Weiß, "Supper H. Forecasting liquidity-adjusted intraday Value-at-Risk with vine copulas," Journal of Banking \& Finance, vol.37, pp. 3334-3350, 2013.

[16] G. B. Fan, Y. Zeng and W. G. Huang, "A multi - asset portfolio risk measure the way: just rattan copula," Quantitative Economic, Technical and Economic Research, vol.1, pp.88-102, 2013.

[17] J. Gao, "Vine Copula model an VaR forecast for multi-asset portfolio," Journal of Applied Statistics and Management, vol.32, pp.247-258, 2013.

[18] B. Z. Zhang, Y. Wei and J. Yu, "The study of correlation and portfolio selection among multi-markets based on EVT-Vine-Copula," Journal of Management Science, vol.05, pp.133-144, 2014.

[19] F. Ma, Y. Wei and D. S. Huang, "Measurement of dynamic stocks portfolio VaR and its forecasting model based on vine copula," Systems Engineering-Theory \& Practice, vol. 35, pp.26-36, 2015.

[20] L.T. Zhao, T. Li and Y. J. Zhang, "Measuring the price risk of energy portfolio with copula-VaR model," System Engineering-Theory \& Practice, vol. 35, pp.771-779, 2015.

[21] A. Sklar,"“Fonctionde repartition a dimension stleurs marges," Publ Inst Stat Univ Paris, 1959, pp.229-231.

[22] E. C. Brechmann, "Truncated and simplified regular vines and their applications," Diploma Thesis: Technische Universitat München, 2010.

[23] P. F. Christoffersen, "Evaluating interval forecasts," International economic review, pp.841-862, 1998. 